\title{
COST-BENEFIT-BASED IMPLEMENTATION STRATEGY FOR GREEN LIGHT OPTIMISED SPEED ADVISORY (GLOSA)
}

\author{
Wolfgang Niebel ${ }^{1}$ \\ ${ }^{1}$ German Aerospace Center (DLR), Institute of Transporation Systems, Department of \\ Traffic Management, Rutherfordstr. 2, 12489 Berlin, Germany \\ wolfgang.niebel@dlr.de
}

\begin{abstract}
GLOSA as a particular application of communicating vehicles and infrastructure has become a technically proved system. The paper shows that the implementation of the GLOSA functionality can generally be recommended from the economical point of view. This result is achieved by applying a simulation study combined with principles of a Cost-Benefit-Analysis to a realworld demonstration site in Braunschweig. However, constraints arise by the complexity of junction signalling and approach lanes.
\end{abstract}

Keywords: Transport economy and policy, Cost-benefit-analysis CBA, Strategies for transport telematics solutions implementation

\section{Introduction and Acknowledgement}

This paper presents possible real-world strategies to implement the cooperative road telematics application GLOSA (Green Light Optimised Speed Advisory). Briefly described the vehicle receives via vehicle-to-infrastructure (V2I) communication the Signal Phase and Timing (SpaT) Data of the traffic signal it is approaching. Applying Advanced Driver Assistance Systems (ADAS) either the driver is informed about the resulting speed optimum or the conventional Automatic Cruise Control (ACC) is directly fed with the corresponding input. Within a few years, GLOSA has become a well-researched and developed technology in the ITS spectrum which aims to avoid or alleviate stops of vehicles at traffic signals in order to reduce fuel consumption, noise, and pollutant emissions, as well as to achieve gains in traffic efficiency.

A lot of studies investigated the possible effects by applying microscopic traffic simulation software. The thereto modelled networks and traffic parameters remain in most of the cases rather simple, e.g., ten uni-directional platooning cars on a single lane [1], or a uni-directional corridor including two traffic lights without any turns, oncoming or crossing traffic [2]. Even the comprehensive investigations in [3] concentrate on a short time period during the peak hour and give results only as an average of all 15 simulated junctions. Common criteria to measure the alterations are fuel consumption and delay or stop time. All sources conclude that the desired improvements can be realized but with a varying magnitude of impact depending on the control parameters. While the maximum fuel gain reaches $30 \%$ under perfect

J. Mikulski (Ed.): TST 2013, CCIS 395, pp. 312-320, 2013 (C) Springer-Verlag Berlin Heidelberg 2013 The final publication is available at http://link.springer.com/chapter/10.1007/978-3-642-41647-7_38 


\section{COST-BENEFIT-BASED IMPLEMENTATION STRATEGY FOR GREEN LIGHT OPTIMISED SPEED ADVISORY (GLOSA)}

conditions [1], realistic values seem to be less than $10 \%$ [2], [3]. But do these figures justify the necessary investments by public road authorities and vehicle owners also in an economical way? And if so, how and where to start with the technology rollout? The German national research project KOLINE went beyond the previous trafficconcentrated studies by adding a junction-related cost-benefit-analysis (CBA) [4] which integrates all different benefits, differentiates between junction types, and therefore supports stakeholders in deciding implementation strategies for the cooperative GLOSA system. Section 2 of this paper describes the traffic simulation and the CBA, followed by the generated CBA results in section 3. The arising implications and hints at further detailed studies to be conducted are contained in section 4.

The research within the KOLINE project received financial support from the German Federal Ministry of Economics and Technology (BMWi) according to a decision of the German Federal Parliament within the 3rd transport research framework "Mobility and Transport Technologies". The Institute of Transportation and Urban Engineering at TU Braunschweig was the leader of this project, of which in particular Dipl.-Ing. Ralf Kutzner contributed to the results' interpretation. Further project partners were the Institut für Automation und Kommunikation e.V. Magdeburg (ifak), Institute of Control Engineering TU Braunschweig, Transver GmbH Munich, and Volkswagen AG Wolfsburg (VW).

\section{Methodology}

\subsection{Network Parameters}

The regarded road network as in Fig. 1 is situated in the north-east of the German city Braunschweig (Brunswick), which is inhabited by roughly 250,000 people. Within this network the speed limit is $50 \mathrm{~km} / \mathrm{h}$. Two out of the three succeeding traffic signal actuated intersections - nodes K61 and K47 - form part of the dual carriageway inner ring road. On workdays these sections bear an average daily traffic (ADT) of more than 36,000 vehicles (sum of both directions according to the ADT definition). The western and eastern intersections, K61 resp. K46, both have their obvious superordinate stream in the east-west- and west-east-direction and perpendicular subordinate approaches. This layout results in a simple 2-phase fixed-time signal program with jointly signalised green times for all three turns of each respective approach. In contrast the middle intersection K47 at the merge of the ring road with the federal road B248 and another major road has remarkable traffic loads on almost all of its turn directions. This leads to a complex 4-phase fixed-time signal program where at each approach green time for left turns begins several seconds later than for the combined straight and right turns. These separately signalised left turns are assigned with dedicated lanes only within a relatively short range of approx. $50 \mathrm{~m}$ before the junction, meaning that left turning cars share most of the combined approach lanes together with straight and right turn traffic.

J. Mikulski (Ed.): TST 2013, CCIS 395, pp. 312-320, 2013 (C) Springer-Verlag Berlin Heidelberg 2013 The final publication is available at http://link.springer.com/chapter/10.1007/978-3-642-41647-7_38 
Thus, it will be distinguished between a simple and a complex junction. To get a better idea of the intersection layouts including their lane-turn-assignments they are depicted in Fig. 2 and Fig. 3.

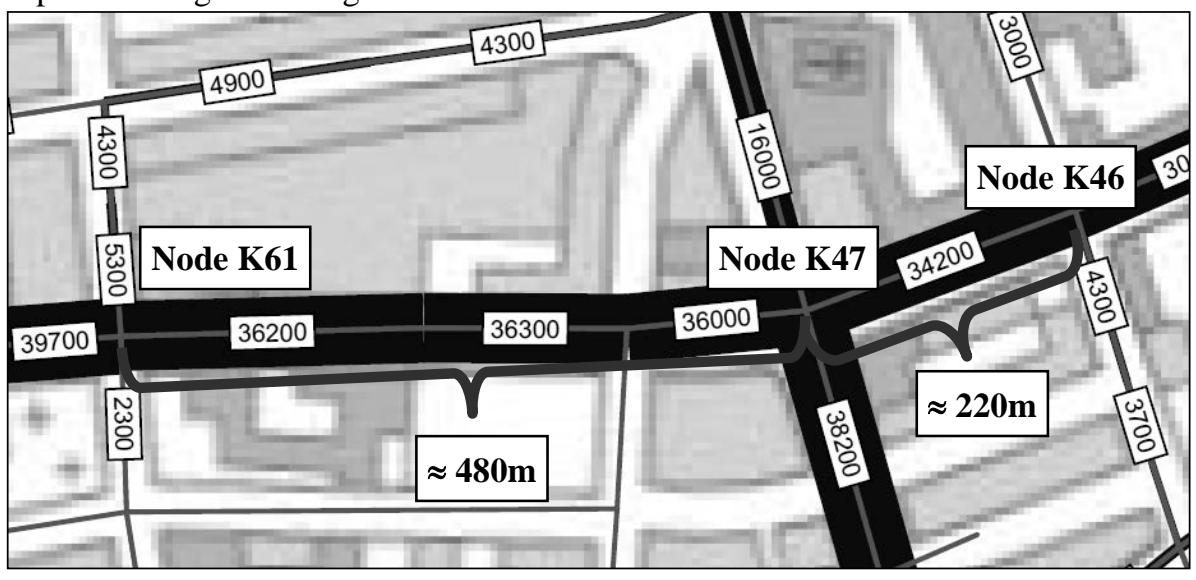

Fig. 1. Simulated road network with position of intersection nodes and ADT on its links 

ADVISORY (GLOSA)

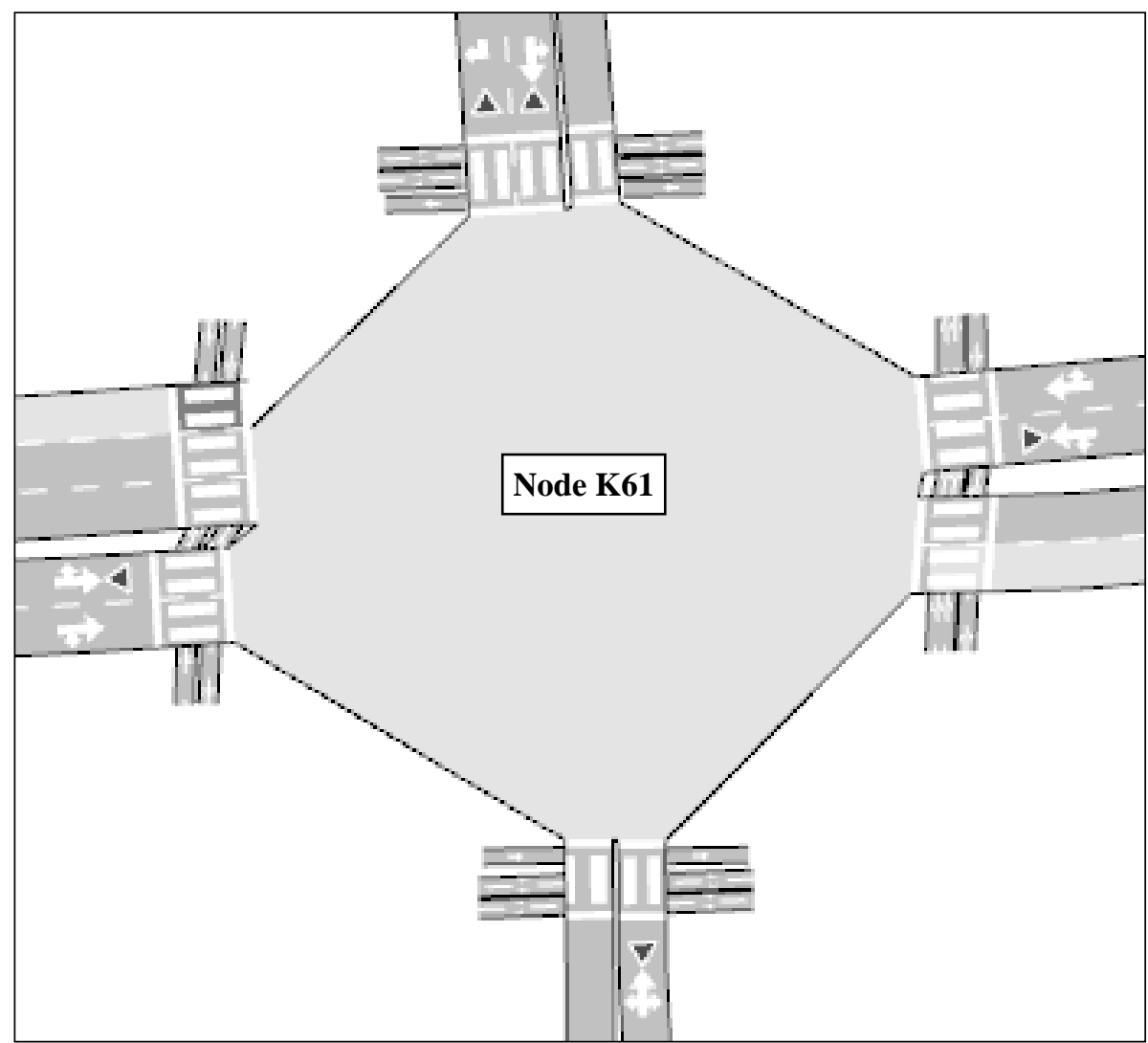

Fig. 2. Detailed lane layout of western simple intersection K61 


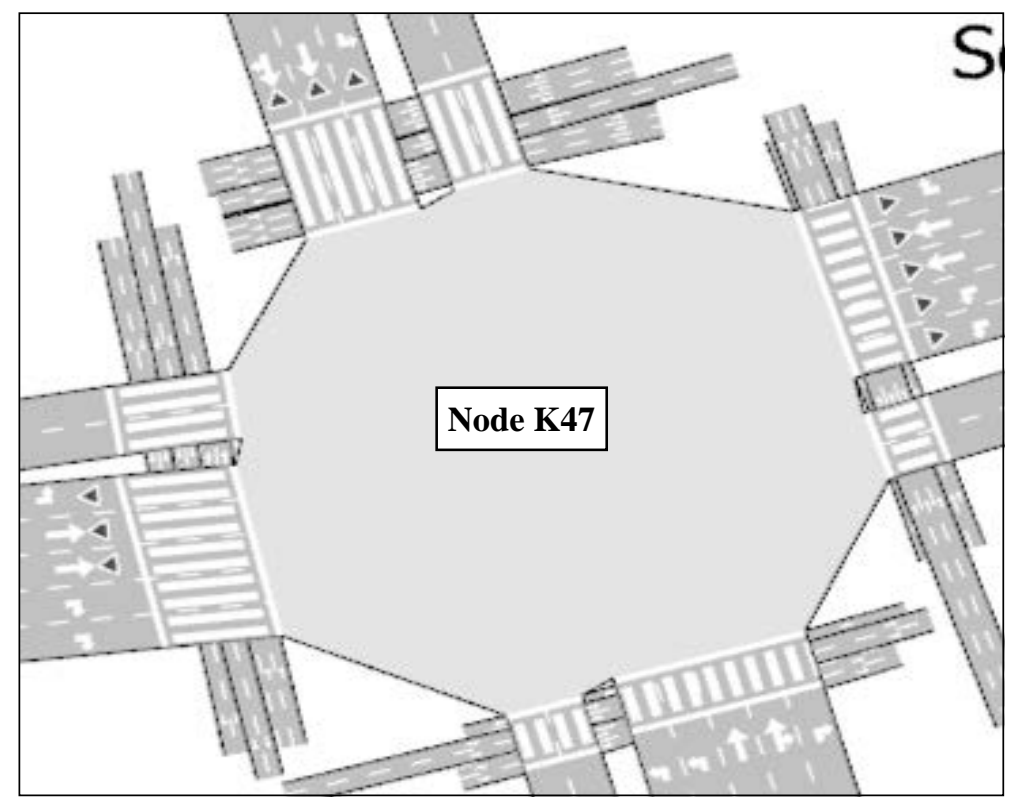

Fig. 3. Detailed lane layout of central complex intersection K47

\subsection{Microscopic Traffic Simulation}

The above described network was modelled with the microscopic simulation software AIMSUN 6.1.3 in time steps of $1 \mathrm{~s}(1 \mathrm{~Hz})$. Implemented traffic load curves between 6 a.m. and 10 p.m. mirror the real ones taken from automatic counting systems. The model calibration on the parameter network travel time allowed a Root Mean Square Error (RMSE) of less than $10 \%$ between the observed real values and the averaged values of the 16 simulation runs. This number was calculated according to the Student's t-distribution. The subsequent model validation on the parameter tailback length yielded an accepted error of $7.2 \%$. The $\mathrm{NO}_{\mathrm{x}}, \mathrm{CO}, \mathrm{HC}$, and $\mathrm{PM}$ pollutant emissions of cars, trucks, and busses were calculated using customised factors derived by the German handbook HBEFA. The applied fuel consumption factors are given in the German evaluation procedure BVWP [6]. Emissions of the climate gas $\mathrm{CO}_{2}$ can directly be derived from these diesel and petrol numbers. Pollutant immission, noise emissions, and accidents were not part of the simulation due to the lack of embedded software algorithms.

The GLOSA functionality is coded outside the actual traffic simulation but acts as software-in-the-loop via an Application Programming Interface (API) by altering the desired speed of equipped vehicles within the $300 \mathrm{~m}$ proximity of a regarded junction. As a model simplification perfect message propagation conditions were assumed without further simulation of communication. The simulation software supported this V2I integration by providing the edible vehicle type attribute penetration rate. The respective control parameter of the scenarios T1 to T6 were set $[0 ; 5 ; 15 ; 25 ; 35] \%$. Additionally, all scenarios comprised a signal program optimisation based on semi- 


\section{COST-BENEFIT-BASED IMPLEMENTATION STRATEGY FOR GREEN LIGHT OPTIMISED SPEED ADVISORY (GLOSA)}

aggregated traffic counts of 15 minute intervals and a consequential volume prediction. Scenarios T2 to T5 also incorporated the broadcast of estimated tailbacks to enhance the calculation accuracy of optimal speeds. These technical aspects are described in detail within [7] and [8].

\subsection{Cost-Benefit-Analysis}

Cost determination. The investment costs per junction $C_{J}$ comprise the communicating Road-Side Unit (RSU) at estimated $C_{R S U}=10,000 €$ and the optimisation software update at $C_{S W}=3,000 €$ for the evaluation period of 20 years. These prices base upon a real offer. The vehicle investment costs per On-Board Unit (OBU) can be assumed at $C_{O B U}=500 €$, the usage duration equals the average vehicle life cycle of 10 years. The Application Unit (AU) being the ADAS/ACC is already existent and thus remains disregarded. The operation and maintenance costs of OBUs and RSUs seem to be negligible - the applied OBU LinkBird-MX of the KOLINE project for example consumes during normal operation only $3 \mathrm{~W}$.

It is important to make further considerations, e.g., how many equipped junctions $n_{J}$ a vehicle passes by during its trip, whether it is riding back and forth during a day (ADT/2), and how many different V2I functionalities are served by the OBU. Thereby only a fraction of these costs are assigned to one particular junction and functionality. In the KOLINE case we assumed that the penetration rate $R_{O B U}=[0 ; 5 ; 15 ; 25 ; 35] \%$ and the number of functions $n F_{O B U}$ are rising at the same rate, that is $\frac{R_{O B U}}{n F_{O B U}}=$ const. $=\frac{5 \%}{1} . . \frac{35 \%}{7}=0.05$. This assumption expresses the rising OBU attractiveness when more and more tasks can be executed with it.

The constant annual costs are calculated without discounting effects due to interest rates far below $3 \%$.

Benefit derivation. Benefits - as difference of a criterion between the base line and a dedicated scenario - were computed for the criteria travel time, vehicle operation incl. fuel consumption, climate gas emission, and pollutant immissions basing on the simulated emissions and an external model. The original unit values were monetised according to the updated official German cost unit rates in [9] and afterwards summed up. The temporal extrapolation of the simulated average workday results first took into consideration the amount of 201 similar workdays per year. Further 101 days p.a. are workdays during school holidays, as well as Saturdays. The achievable benefits were linearly scaled down with the same factor the ADT decreases during these days. This rough and arguable approach was applied again to the remaining Sundays and public holidays.

Cost-Benefit-Figures. The annual benefits and costs can be put into relation either by their Benefit-Cost-Ratio (BCR) which should be greater than 1.0, or by their difference (Net Present Value NPV) which should be greater than zero to make the valuated project economically worth.

J. Mikulski (Ed.): TST 2013, CCIS 395, pp. 312-320, 2013 (C) Springer-Verlag Berlin Heidelberg 2013

The final publication is available at http://link.springer.com/chapter/10.1007/978-3-642-41647-7_38 


\section{Results}

\subsection{Costs}

The constant annual costs of scenario $\mathrm{T} 1$ with $0 \%$ penetration rate amount to exceptional low $150 €$ per junction. The costs of scenarios T2 to T6 can be calculated under the aforementioned assumptions as can be seen in Fig. 4. It becomes clear, that the number of equipped junctions a vehicle is traversing on its journey plays a significant role. The complexity has no direct influence on the costs, but high ADT volumes normally yield more complex junctions in reverse.

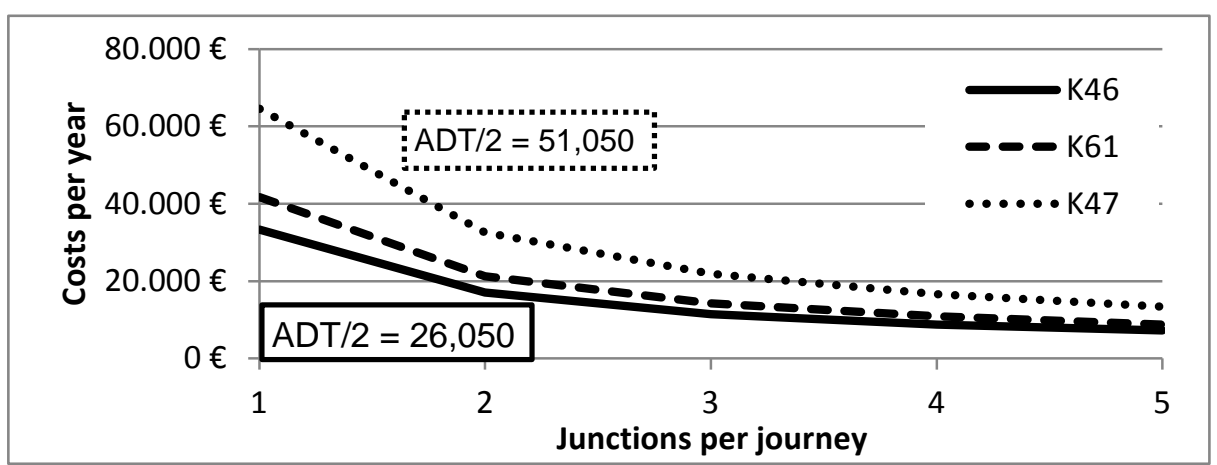

Fig. 4. Annual costs per junction

\subsection{Benefits}

The overall benefit of each junction can be seen in Fig. 5 .

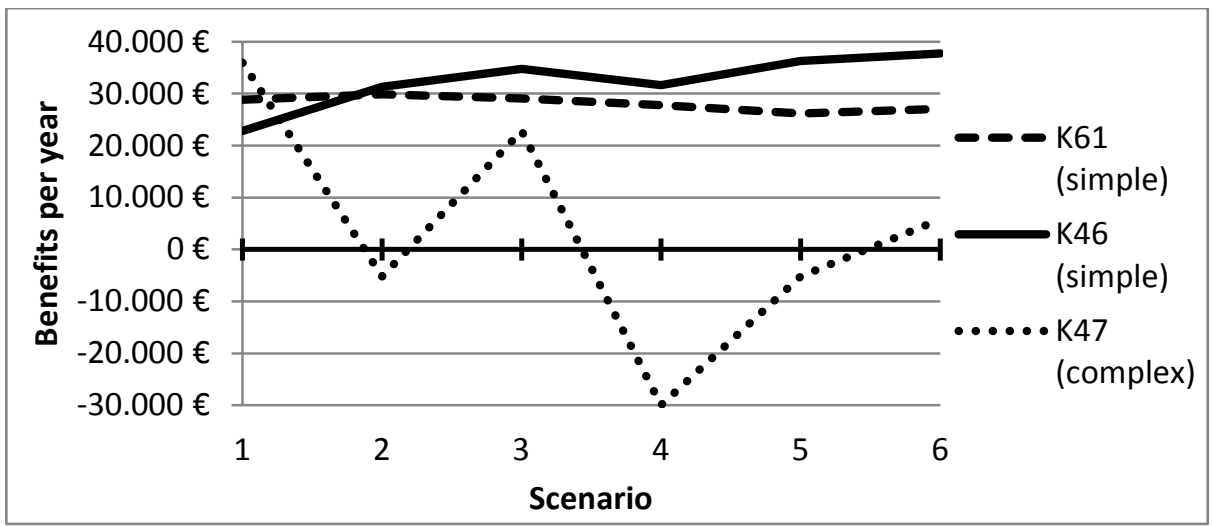

Fig. 5. Annual benefits per junction 


\section{COST-BENEFIT-BASED IMPLEMENTATION STRATEGY FOR GREEN LIGHT OPTIMISED SPEED ADVISORY (GLOSA)}

It is notable that the complex junction K47 has negative benefits in some scenarios and a general decline of benefit with rising penetration rate. The highest benefit results from the pure signal program optimisation in scenario T1. This can be explained by the geometry of the approaching lanes (cf. sec. 2.1), where the separately signalised left turns share the combined approach lanes the first $250 \mathrm{~m}$ of the cooperate message broadcast radius together with straight and right turn traffic. Due to the shifted green time beginnings the optimal speed of some vehicles is lower than for others causing unwanted slow-downs of following vehicles. Thus the traffic flow of the status quo can become worse with the V2I application.

Both simple junctions do not face this problem and therefore have a positive benefit in all scenarios. Nevertheless, there is no continuous functional relation between the rate and benefit. The fall-back of scenario T4 remains unexplained.

At each junction scenario T6 at a penetration rate of $35 \%$ and without the tailback broadcast is better than scenario $\mathrm{T} 5$ at the same rate but with the tailback incorporation. This can be explained by unsuitable algorithms.

\subsection{Cost-Benefit-Figures}

The following Table 1 incorporates only costs and benefits for the two simple junctions due to the negative effects of the complex junction. The BCR shows again the outstanding performance of scenario T1 without any communication units. But also the other scenarios perform well when the equipped vehicles traverse more than one cooperative junction per journey. In Braunschweig, e.g., the average number is 2.5. The NPV of scenario T1 is only achievable with a penetration rate of at least $35 \%$ and six traversed junctions per journey.

Table 1: Benefit-Cost-Ratios and absolute net values (in italic) per scenario

\begin{tabular}{l|c|c|c|c|c}
\hline Scenario & \multicolumn{5}{c}{ Number of traversed junctions $\boldsymbol{n}_{\boldsymbol{J}}$} \\
& 1 & 2 & 3 & 4 & 5 \\
\hline $\mathrm{T} 1$ & \multicolumn{5}{c}{$172 / 51,300 €$} \\
$\mathrm{~T} 2$ & 0,8 & 1,6 & 2,4 & 3,1 & 3,8 \\
& $-13.800 €$ & $23.000 €$ & $35.400 €$ & $41.500 €$ & $45.200 €$ \\
$\mathrm{~T} 3$ & 0,9 & 1,7 & 2,5 & 3,2 & 4,0 \\
& $-11.100 €$ & $25.700 €$ & $38.100 €$ & $44.200 €$ & $47.900 €$ \\
$\mathrm{~T} 4$ & 0,8 & 1,6 & 2,3 & 3,0 & 3,7 \\
& $-15.600 €$ & $21.200 €$ & $33.600 €$ & $39.700 €$ & $43.400 €$ \\
$\mathrm{~T} 5$ & 0,8 & 1,6 & 2,4 & 3,2 & 3,9 \\
& $-12.500 €$ & $24.300 €$ & $36.700 €$ & $42.800 €$ & $46.500 €$ \\
$\mathrm{~T} 6$ & 0,9 & 1,7 & 2,5 & 3,3 & 4,1 \\
& $-10.200 €$ & $26.600 €$ & $39.000 €$ & $45.100 €$ & $48.800 €$ \\
\hline
\end{tabular}

J. Mikulski (Ed.): TST 2013, CCIS 395, pp. 312-320, 2013 (C) Springer-Verlag Berlin Heidelberg 2013

The final publication is available at http://link.springer.com/chapter/10.1007/978-3-642-41647-7_38 


\section{Implications and Further Studies}

The introduction of the GLOSA functionality can generally be recommended from the economical point of view. As shown in sec. 3.2 constraints arise by the complexity of junction signalling and approach lanes. It must be ensured that differently signalised turn flows will not overlap and thereby influence each other in a negative way. As long as such effects cannot be excluded neither by the junction geometry nor by enhanced V2X technology GLOSA should be considered only for simple junctions. Further studies are needed for these extended V2I or V2V systems.

Since rising penetration rates seem to have only marginal positive effects, if at all, the speed of equipping the vehicle fleet is unimportant. In contrast, the number of traversed junctions per journey plays an important role. More investigations are needed which vehicle groups exceptionally often pass traffic signals, but it is likely that beside public transport busses commercial purpose vehicles like taxis or delivery vans are amongst them. They might also traverse a particular traffic signal more than twice a day and hence improve the Benefit-Cost-Rate further. Therefore, the market segment of commercial and fleet vehicles with a high kilometrage should be addressed first to be equipped with the GLOSA system.

\section{References}

1. Sanchez, M., Cano, J.-C., Dongkyun Kim: Predicting Traffic lights to Improve Urban Traffic Fuel Consumption. In: 6th International Conference on ITS Telecommunications Proceedings, pp. 331-336. IEEE (2006), DOI: 10.1109/ITST.2006.288906

2. Katsaros, K., Kernchen, R., Dianati, M., Rieck, D.: Performance study of a Green Light Optimized Speed Advisory (GLOSA) Application Using an Integrated Cooperative ITS Simulation Platform. In: 7th IEEE International Wireless Communications and Mobile Computing Conference (IWCMC), pp. 918-923. IEEE, New York (2011)

3. Tielert, T., Killat, M., Hartenstein, H., Luz, R., Hausberger, S., Benz, T.: The impact of traffic-light-to-vehicle communication on fuel consumption and emissions. In: Internet of Things (IOT 2010), pp. 1-8. IEEE (2010), DOI: 10.1109/IOT.2010.5678454

4. Niebel, W., Bley, O., Ebendt, R.: Evaluation of Microsimulated Traffic Light Optimisation Using V2I Technology. In: Communications in Computer and Information Science, 329, pp. 18-25. Springer Berlin Heidelberg (2012). DOI: 10.1007/978-3-642-34050-5

6. Bundesministerium für Verkehr, Bau- und Wohnungswesen (publisher): Bundesverkehrswegeplan 2003, Grundlagen für die Zukunft der Mobilität in Deutschland. Beschluss der Bundesregierung vom 2. Juli 2003, Berlin (2003)

7. Naumann, S. and O. Bley (2012). A System for Traffic Light Control Optimisation and Automated Vehicle Guidance Using the V2X Communication Technology. In: Archives of Transport Systems Telematics, Vol. 5, pp. 20-23. Polish Association of Transport Telematics.

8. Bley, O., R. Kutzner, B. Friedrich and T. Schüler (2012). Improvement in Traffic State Estimation at Signal Controlled Intersections by Merging Induction Loop Data with V2X Data. In: Archives of Transport Systems Telematics, Vol. 5, pp. 3-7. Polish Association of Transport Telematics.

9. Bundesministerium für Verkehr, Bau und Stadtentwicklung BMVBS (publisher): Aktualisierung von Bewertungsansätzen für Wirtschaftlichkeitsuntersuchungen in der Bundesverkehrswegeplanung, Final Report. Essen, Freiburg, München (2009) 